\title{
Complete Heart Block and Acute Inferior Myocardial Infarction Due to
}

\section{Generalized Vasospasm}

\author{
Yahya Kemal Icen ${ }^{1 *}$, Mevlut Koc ${ }^{1}$
}

\begin{abstract}
Objective: Sixtyone years old female patient had applied to emergency department with chest pain. Her ECG taken was compliant with acute inferior myocardial infarction and complete heart block. In her coronary angiography (CAG), there was generalized vasospasm from osteal in left anterior descending coronary artery (LAD) and circumflex artery (CX). $10 \mathrm{mcg} / \mathrm{kg} / \mathrm{mn}$ nitrate was infused in the coronary and vasospasm is disappeared. After CAG, her ECG was normal and she discharged with medical treatment recommendations
\end{abstract}

Keywords: Complete Heart Block, Generalized Vasospasm, Coronary

\section{Introduction}

The spasm in coronary arteries may cause a wide spectrum of cardiac cases from temporary chest pain to acute ST elevation myocardial infarction (STEMI)1. Despite vasospasm is usually resolved with nitrate and nitrate derivatives, sometimes cardiologists may encounter some refractory cases 2,3 . If the vessels with vasospasm feed the sinoatrial or atrioventricular nodes, brady-arhythmic complications may develop in the patient4. In this case, we have submitted a patient with complete heart block due to generalized vasospasm.

Case: the 61 years old female patient had applied to emergency department with retrosternal chest pain. Hypertension and smoking history were present in her history. In the CAG of the patient applied one month before, the presence non-critical lesions were reported. In her physical examination, there were no finding except the pulse of 35 pulse/minute. In the ECG taken; ST segment elevation and complete heart block were present at D2, D3, AVF derivations (figure 1). The patient was diagnosed as acute inferior myocardial infarction and complete heart block and she was hospitalized at coronary intensive care unit (CICU). Due to the non-critical obstructions in the previous CAG, vasospasm was considered and nitrate infusion at a dose of 5 microgram/kilogram/minute was started. In the assessment of echocardiography, inferior wall was observed as hypokinetic and ejection fraction was measured as $55 \%$. The patient, whose general status was impaired, was taken to the CAG unit.
In the CAG result, it was found that there was a left anterior descending artery (LAD) and circumflex artery (CX) was completely obstructed from osteal (figure 2) and there were plaques in the right coronary artery (RCA) (figure 3). Nitrate dosage was increased to $10 \mathrm{mcg} / \mathrm{kg} / \mathrm{minute}$ then the completely occluded regions in LAD and CX had opened (figure 4). She was taken to the CICU and in her ECG taken, it was observed the ST segment elevation had disappeared and it was at the sinus rhythm (figure 5). The patient, who was monitored at CICU for one day and at cardiology service for 2 days, was discharged with nitrate, diltiazem, acetylsalicylic acid and atorvastatine. Drug treatment was continued at her outpatient polyclinic follow-ups.

\section{Discussion}

In the ECG of our patient, despite the ST elevation in inferior derivations and a complete heart block, we considered vasospasm due to the non-critical obstructions in the previous CAG one month ago and thus, we did not apply CAG instantly. Firstly, we had waited to have vasospasm dissolve via nitrate infusion at CICU. In the literature; it is specified that a female patient had a recurrent completely heart block due to coronary vasospasm and thus a permanent pacemaker was placed. In another patient, as a result of spontaneously developed generalized vasospasm; the development of complete heart block, cardiogenic shock, ventricular fibrillation and ischemic stroke was mentioned.

Received 05-01-2018 Accepted 17-01-2018 Available Online 31-01-2018

1 Health Science University Adana Health Research and Application Center Cardiology Department, Adana, TR

* Corresponding Author: Yahya Kemal Icen E-mail: dryahyakemalicen@ gmail.com Phone: +90 5437720025 
In a series of three cases; DDD-R permanent pacemaker with an intracardiac defibrillator (ICD) was placed to the patients at whom high-grade heart block and cardiac arrest occurred due to vasospasm7. Again in another case, a permanent pacemaker was placed due to bradyarrhythmia caused by vasospasm8.

In our patient, the nitrate dosage given at CICU was not quite efficient. We have taken out patient to CAG unit due to the impairment in her general status. As a result of intra-coronary nitrate infusion, vasospasm was dissolved..
The patient was discharged with medical recommendations. Contrary to most of the patients in the literature, there was no need for a permanent pacemaker in her outpatient follow-ups

\section{Conclusion}

In coronary vessels, general vasospasm can cause acute inferior myocardial infarction and complete heart block. In these patients, vasodilatory therapy may be tried before invasive treatment.

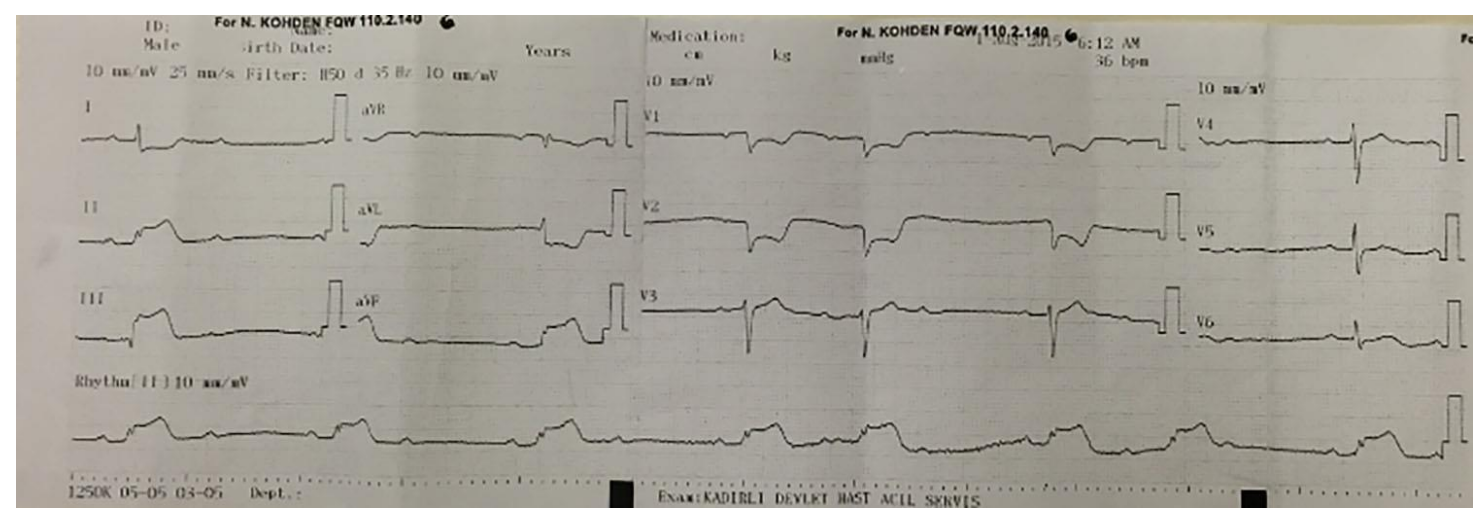

Figure 1: Complete heart block and acute inferior myocardial infarction in 12 lead surface ECG

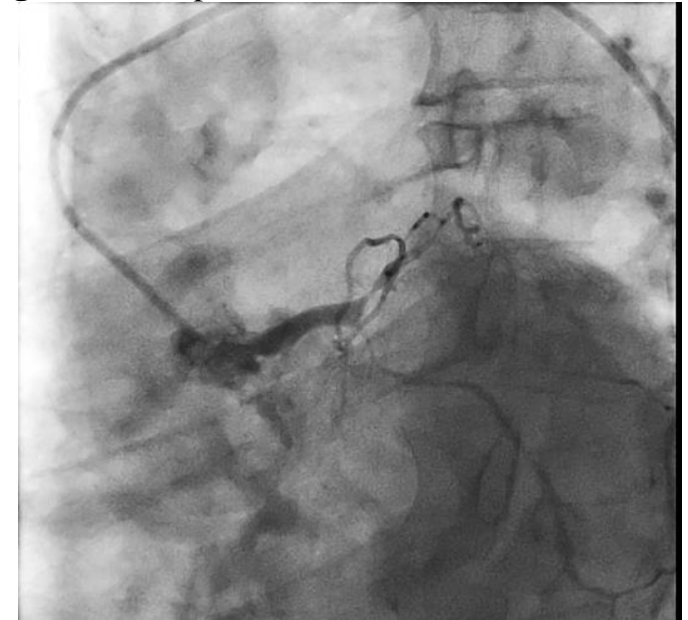

Figure 2: Generalize vasospasm in LAD and CX

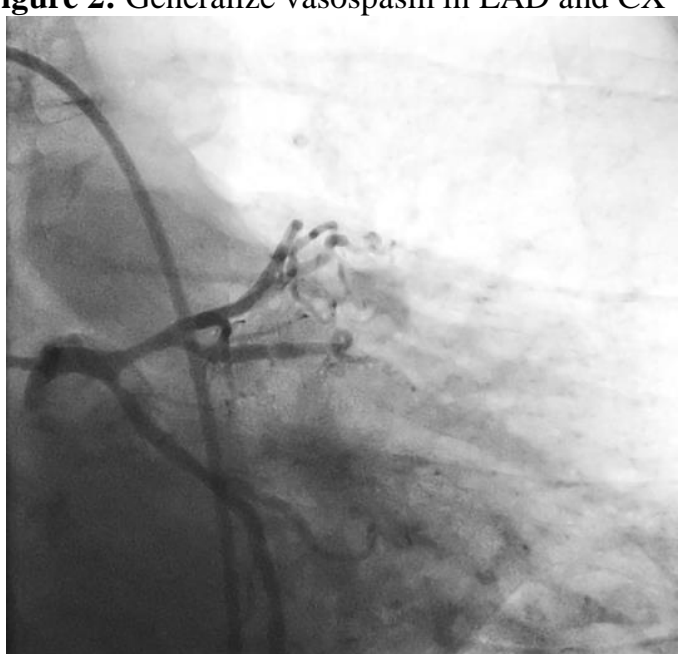

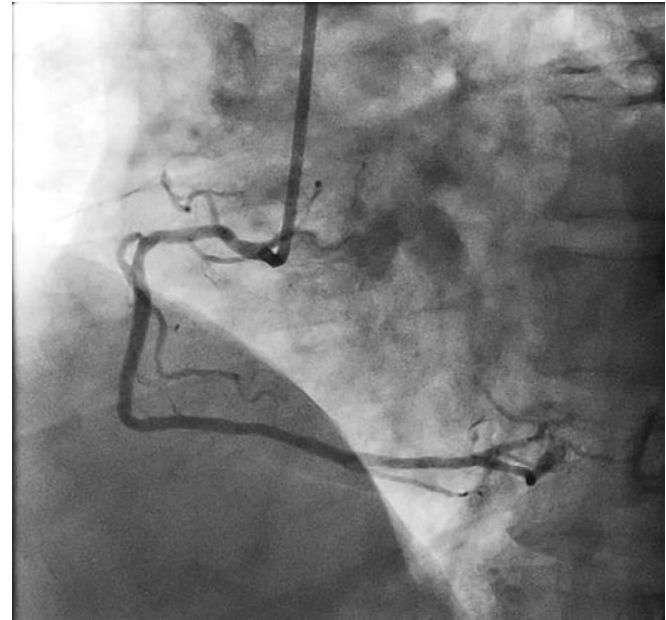

Figure 3: Non-critical plaque in RCA

Figure 4: Resolving vasospasm after $10 \mathrm{mcg} / \mathrm{kg} / \mathrm{minute}$ nitrate infusion 


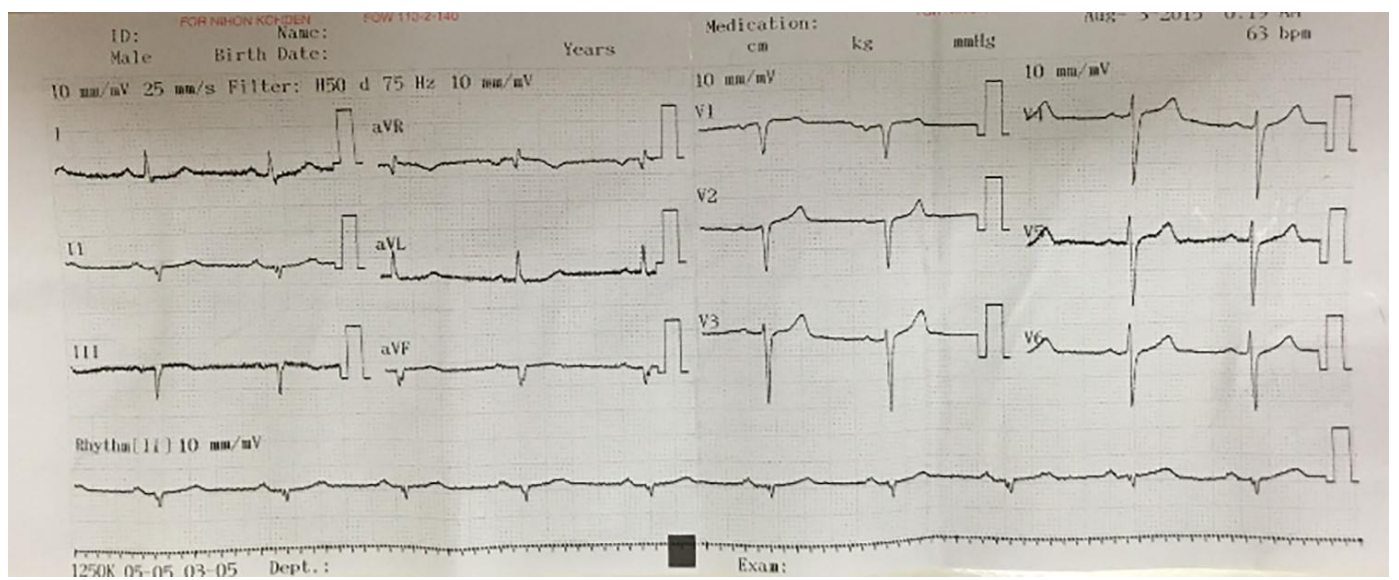

Figure 5: Resolution ST elevation and sinus rhythm in 12 lead surface ECG

\section{Acknowledgments, Funding: None}

Conflict of Interest: The authors declare no potential conflicts of interest with respect to the research, authorship, and/or publication of this article.

Author's Contributions: YKI, MK: Research concept and design; Patients examination Procedures, data collecting, biochemical analysis and interpretation of data. All authors approved the final version of the manuscript,

Ethical issues: All Authors declare that Originality of research/article etc... and ethical approval of research, and responsibilities of research against local ethics commission are under the Authors responsibilities. The study was conducted due to defined rules by the Local Ethics Commission guidelines and audits.

\section{References}

1. Hirofumi Yasue, Hitoshi Nakagawa, Teruhiko Itoh, Eisaku Harada, Yuji Mizuno. Coronary artery spasm-Clinical features, diagnosis, pathogenesis, and treatment. Journal of Cardiology 2008;51: 2-17.

2. JCS Joint Working Group. Guidelines for diagnosis and treatment of patients with vasospastic angina (Coronary Spastic Angina) (JCS 2013). Circ J. 2014;78:2779-801.

3. Kusama Y, Kodani E, Nakagomi A, et al. Variant angina and coronary artery spasm: a the clinical spectrum, pathophysiology, and management. J Nippon Med Sch 2011;78:4-12

4. Ledakowicz-Polak A, Ptaszynski P, Polak L, et al. Prinzmetal's variant angina associated with severe heart rhythm disturbances and syncope: a therapeutic dilemma. Cardiol J. 2009;16:269-72.

5. Filipa Ferreira, Lorette Cardona, Bruno Valente, João Abreu, Eduardo Antunes, Lurdes Ferreira, José Alberto Oliveira, Rui Ferreira. High-degree atrioventricular block induced by Prinzmetal angına. Rev Port Cardiol. 2012;31:233-235

6. Viji S. Thomson, Osama Tariq, and Hafidh Al Hadhi. Malignant Multivessel Coronary Spasm Complicated by Myocardial Infarction, Transient Complete Heart Block, Ventricular Fibrillation, Cardiogenic Shock and Ischemic Stroke. Oman Medical Journal 2014; 4:296-298

7. Seniuk W, Mularek-Kubzdela T, Grygier M, Grajek S, Cieśliński A. Cardiac arrest related to coronary spasm in patients with variant angina: a three-case study. J Intern Med. $2002 ; 252: 368-76$

8. Siliste RN, Savulescu-Fiedler I, Siliste C. Bradyarrhythmic syncope in a patient with Prinzmetal's variant angina: a case report. American Journal of Emergency Medicine 2013; 996.e1-996.e4

Copyright $\odot 2018$ The Author(s); This is an open-access article distributed under the terms of the Creative Commons Attribution License (http://creativecommons.org/licenses/by/4.0), which permits unrestricted use, distribution, and reproduction in any medium, provided the original work is properly cited. All Rights reserved by international journal of Medical Science and Discovery. 